Boise State University

ScholarWorks

$4-2020$

\title{
Duck Valley Reservation (Owyhee, Nevada)
}

Tim Thornes

Boise State University 


\title{
DUCK VALLEY RESERVATION (OWYHEE, NEVADA)
}

\author{
TIM THORNES
}

Boise State University

The Shoshone-Paiute community of Duck Valley includes reservation territory that straddles the Idaho-Nevada border. Recordings of the Duck Valley communolect were made at the home of the speaker's niece in the presence of several extended family members, including speakers, passive bilinguals and nonspeakers alike, as well as children. The speaker was 60 years old at the time and clearly enjoyed recounting Coyote stories. That said, a few of the stories were considered by her family to be a bit too lurid to include here.

\section{COYOTE AND BEAVER ${ }^{1}$}

\section{$\dagger$ Phyllis Astarloa, Narrator}

In this story, the emphasis, according to the speaker, is on the consequences of lying - something Coyote, of course, is known for. The story is somewhat incomplete, by the admission of the speaker, and exhibits expressions of uncertainty, inference, and other interesting features of metanarration. The orthographic convention is the same as that adapted for Burns.

(1) Seme yaisi su idza'a tewau mi'i idza'a unaa,

simi yaisi su=idza?a tiwau mi?i idza?a una one then NOM=Coyote again QUOT Coyote out.there

'Once again, the Coyote, it is said, Coyote out there,'

(2) me idza'a ka pemitu punni, mino'o pemitu punnisi ka unaa miana,

$$
\begin{aligned}
& \mathrm{mi}=\mathrm{idza} \text { ?a } \mathrm{ka}=\text { pimi-tu punni mino?o pimi-tu } \\
& \mathrm{PL}=\text { coyote } \mathrm{ACC}=\text { they }-\mathrm{LOC} \text { see now they-LOC } \\
& \text { punni-si } \mathrm{ka}=\text { una mia-na } \\
& \text { see-SEQ ACC }=\text { out.there go-PTCP }
\end{aligned}
$$

'as when you see coyotes going along, now, yonder going,'

(3) himma ikwinachodamina, ikwitachoda tui hannotui miaugaa.

himma ikwi-načoda-mina ikwi-tačoda tui hanno=tui

what smell-track-go.along smell-track any where $=$ any

${ }^{1}$ Recorded August 25, 1999, Duck Valley Indian Reservation, Owyhee, Nevada 


$$
\begin{aligned}
& \text { miau-gaa } \\
& \text { go-away }
\end{aligned}
$$

'tracking something by scent, tracking (nose down) while going along just anywhere.'

(4) Uu ka manigyana yaisi.

uu ka=mani-gya-na yaisi

like.so $\mathrm{ACC}=$ do-away-PTCP then

'So, that's what he was doing.'

(5) Una ka huude kimaba pideugaa mi, una pideugaa.

$\begin{array}{lllll}\text { una } & \text { ka=huu-di } & \text { kimaba } & \text { pidi-u-gaa } & \text { mii } \\ \text { out.there } & \text { ACC }=\text { flow-NMLZ } & \text { beside } & \text { arrive-PFV-away } & \text { QUOT } \\ \text { una } & \text { pidi-u-gaa } & & & \\ \text { out.there } & \text { arrive-PFV-away } & & & \end{array}$

'So where the water was coming, they say, that's where he arrived.'

(6) Unau chaisi tenikwehewene mii.

una-u čaisi tinikwihi-wini mii

out.there-FOC then sing-PROG:SG QUOT

'And that's where he was singing, it is said.'

(7) Usu . . hii gya. . . Hayu usu nani’a?

u-su hii=gya hayu u-su na-ni?a

$3 \mathrm{SG}-\mathrm{NOM}$ thing $=\mathrm{MOD}$ how 3SG-NOM MID-call

'What was that ... What is it called?'

(8) Beaver? What do you call "beaver"?

(9) Ha’ngiisa!

ha?piisa

beaver

'Beaver!'

(10) Ha'ngisa kobaba kemaba'yuna, hipe himmaga huupi himmaga watti,

$\begin{array}{lccl}\text { ha?yisa } & \text { koba-ba } & \text { kimaba-Pyu-na } & \text { hipi } \\ \text { beaver } & \text { face-by } & \text { beside-from-PTCP } & \text { thing } \\ \text { himma=ga } & \text { huupi } & \text { himma=ga } & \text { watti } \\ \text { what=MOD } & \text { stick } & \text { what=MOD } & \text { search:DUR }\end{array}$

'Next to the beaver's face, looking for a stick or something,'

(11) yah, himma e tenikwehewenenemi.

yah himma $\dot{i}=$ tinikwihi-wini-nimi

yes what 2SG=sing-PROG:SG-RNDM:SG

'continuing to sing walking about.' 
(12) Ha'ngisa ka tenikwehewene.

haPyiisa $\mathrm{ka}=$ tinikwihi-wini

Beaver $\quad \mathrm{ACC}=$ sing $-\mathrm{PROG}: \mathrm{SG}$

'So the beaver was singing.'

(13) Himma sa'a 'yu'yuhu puni, himma sa'a 'yu'yuhu punigyaina mi oo.

$\begin{array}{clll}\text { himma=saPa } & \text { Pyu Pyuhu } & \text { puni } & \text { himma=sa?a } \\ \text { what=MOD } & \text { PL fat } & \text { see } & \text { what=MOD } \\ \text { Pyu Pyuhu } & \text { puni-gyai-na } & \text { mii } & \text { oo } \\ \text { PL fat } & \text { see-HAB-PTCP } & \text { QUOT } & \text { DEM }\end{array}$

'Looking for something fat, carefully searching for something fat, (they) say so.'

(14) Idza'a u nakkau, ka ene idza'ana.

idzaPa $\mathrm{u}=$ nakka $-\mathrm{u} \quad \mathrm{ka}=$ ini $\quad$ idza?a-na

Coyote $3 \mathrm{SG}: \mathrm{ACC}=$ hear $-\mathrm{PFV} \quad$ ACC $=\mathrm{INT} \quad$ coyote-PTCP

'The Coyote heard him, because that's how coyotes really are!'

(15) “Ah, hau e manakwi?" mi.

ah hau ii manakwi mii

ah how you act:DUR QUOT

“"So, what are you doing there?" (he) asked.'

(16) "Kai ne yaa, sekwimiakainemi," mii gya sa'a ha'ngisa.

kai nii yaa sikwi-mia-kai-nimi

NEG I here only-go-ABOUT-RNDM:SG

$\mathrm{mii}=\mathrm{gya}=\mathrm{sa}$ ?a ha?pisa

QUOT $=$ MOD $=$ MOD Beaver

" "I'm not doing a thing, just going along," so that Beaver must have been saying.'

(17) “Oo himma e kwattina?" mii.

oo himma $\dot{i}=$ kwatti-na mii

DEM what $2 \mathrm{SG}=$ search-PTCP QUOT

“"So, what are you looking for?" saying.'

(18) "Kai du ne himma. E chaisi hayu?"

kai du nii himma ii čaisi hayu

NEG also I what you then how

، "I'm not (doing) a thing. And you?",

(19) [Teekwi-] u tenikwehe chaisi, eetu, nagedza pisa'yu eniwene mi.

$\mathrm{u}=$ tinikwihi čaisi $\ddot{\mathrm{ii}}-\mathrm{tu}$ nagidza pisa-Pyu ini-wini

3SG:ACC $=$ sing then you-LOC truly good-NOM say-PROG 
mii

QUOT

“"What are you singing along that sounds so good?" (Coyote) said.'

(20) "Kai, himma sokona inate wattina, himma sa kadu'u watti," mi.

$\begin{array}{clll}\text { kai himma } & \text { soko-na } & \text { ina-ti } & \text { watti-na } \\ \text { NEG what } & \text { go.afoot-PTCP here-AT } & \text { search-PTCP } \\ \text { himma=sa } & \text { kaduPu } & \text { watti } & \text { mii } \\ \text { what=MOD } & \text { gone } & \text { search:DUR } & \text { QUOT }\end{array}$

، "So, what should I be looking for in this spot?" saying.'

(21) “Kai! Kai nana - nagedza pisa enikima," mi.

kai kai nagidza pisa ini-kima mii

NEG NEG truly good say-come QUOT

“ "No, indeed it sounds too good (better than you say)!" saying.'

(22) Ha'ngisa kai u teekwi.

ha?nisa kai $\mathrm{u}=$ tiikwi?i

Beaver NEG 3SG:ACC $=$ tell

'Beaver didn't tell him.'

(23) Yaisi, idza'a setahu, idza'a setahu, miu.

yaisi idza?a sita-hu idza?a sita-hu mi-u

then Coyote bad-PFV Coyote bad-PFV QUOT-FOC

'And so Coyote got mad, Coyote got mad, it is said.'

(24) Himma sa'a yuyuhugena, himma sa'a yuyuhugena?

himma $=$ saPa $\quad$ yu-yuhu - gi-na $\quad$ himma $=$ saPa

what $=$ MOD RE-fat-toward-PTCP what $=$ MOD yu yuhu-gi-na

DISTR fat-toward-PTCP

" "So, where's the fat! Where's the fat?",

(25) "Miu ha e?" mi.

mii-u=ha ii mii

QUOT-FOC $=$ Q you QUOT

“"Is that what you're saying/thinking?" said.'

(26) Oono yaisi gya, ha'ngisa u deegweu, “aha, uu manina,” mi.

oono yaisi $=$ gya haPyisa $u=$ ditgwi-u

at.the.time then $=$ MOD Beaver $3 \mathrm{SG}: \mathrm{ACC}=$ tell $-\mathrm{PFV}$

aha uu mani-na mii

yes like.so do-PTCP QUOT

'So, therefore, the Beaver told him, "Yes, that's what I am doing," said.' 
(27) Oo ka yaisi ka kutsu sii unate ga te sayana ka paawaitu ka woisana pisa.

oo ka=yaisi ka=kutsu sii

DEM $\quad \mathrm{ACC}=$ then $\quad \mathrm{ACC}=\mathrm{cow}$ stomach

una $-\mathrm{t} \mathrm{i}=$ ga $\quad \mathrm{t} i=$ sayana

there $-\mathrm{LOC}=\mathrm{MOD} \quad \mathrm{LOGO}=$ guts

$\mathrm{ka}=$ paa-wai-tu $\quad \mathrm{ka}=$ woisa-na pisa

$\mathrm{ACC}=$ water-AREA-to $\mathrm{ACC}=$ wash $-\mathrm{PTCP}$ good

'So, then, the cow's belly, ${ }^{2}$ inside (there), (he) took it and washed it somewhere in the water very well.'

(28) Hau ga yaisi una uka huupi watti, miausi.

$$
\begin{aligned}
& \text { hau =ga yaisi una u-ka huupi watti } \\
& \text { how }=\text { MOD then there 3SG-ACC stick search } \\
& \text { mia-u-si } \\
& \text { go-PFV-SEQ }
\end{aligned}
$$

'So, it must have been as he went to look for the stick.' 3

(29) Ka te chuhugaana oisu 'ma'wiukaasi.

$$
\begin{array}{lll}
\mathrm{ka}=\mathrm{ti}=\mathrm{c} u h u-\text { gaa-na } & \text { oi-su } & \text { Pma?wi-u-kaa-si } \\
\mathrm{ACC}=\mathrm{LOGO}=\text { fat-have-PTCP } & \text { DEM-EMPH } & \text { leave-PFV-away-SEQ }
\end{array}
$$

'The fat he had collected, he left there.'

(30) Me patunapa ga peno'o oi'yu koohau, u sayana ka kadu'u 'mani [yai]si u su'au.

$$
\begin{array}{clll}
\mathrm{mi}=\text { patunapa }=\text { ga } & \text { pino?o } & \text { oi-Pyu } & \text { kooha-u } \\
\mathrm{PL}=\text { intestine=MOD } & \text { as.well } & \text { DEM-from } & \text { pick.up-PFV } \\
\mathrm{u}=\text { sayana } & \mathrm{ka}=\text { kadu } \mathrm{Pu} & \text { Pmani } & \\
\begin{array}{c}
\text { 3SG.POSS=guts } \\
\text { yaisi }
\end{array} & \mathrm{ACC}=\text { gone } & \text { do } & \\
\text { then } & \mathrm{u}=\text { su Pa-u } & &
\end{array}
$$

'So, they also came and got it, the guts, and left; and there was nothing left, having gone and consumed all of it.'

(31) Usu yaisi oi pittegaasi, u sayana kadu'u.

u-su yaisi oi pitti-gaa-si $\mathrm{u}=$ sayana kadu?u

3SG-NOM then DEM arrive-away-SEQ 3SG.POSS=guts gone

'When he got over there, the sayana was all gone.'

\footnotetext{
${ }^{2}$ Translator was uncertain of the exact body part reference here for the term sayana.

${ }^{3}$ A side comment by the speaker.
} 
(32) Oono ka ha'ngisa peno'o ka idza'a settakiu, u wadzi susagwaipeni, ka hayu niitama ga.

$$
\begin{array}{lll}
\text { oono } & \mathrm{ka}=\text { ha?pisa pino?o } & \mathrm{ka}=\mathrm{idza} \text { Pa } \\
\text { at.the.time } \mathrm{ACC}=\text { beaver as.well } & \mathrm{ACC}=\text { Coyote } \\
\text { sitta-ki-u } & \mathrm{u}=\text { wadzi } & \text { su-sagwai-pini } \\
\text { bad-toward-PFV } & \text { 3SG:ACC=hide } & \text { DESID-angry-PROG } \\
\text { kai hayu nitama=ga } & \\
\text { NEG how tell=MOD } &
\end{array}
$$

'And then the Beaver got angry with Coyote, but kept his anger hidden and didn't let on'

(33) Oo chaisi, oo teka tewawa nazu'akeu.

oo čaisi, oo tika tiwawa na-zu Pa-ki-u

DEM then DEM eat collect MID-consume-APPL-PFV

'So then, all that he had gathered was eaten up.'

(34) Oo ka yaisi issayaino oo pe kima'yusu oi'yu koohau.

$$
\begin{array}{clll}
\text { oo ka=yaisi issayai-no } & \text { oo } & \\
\text { DEM ACC=then lie-TEMP } & \text { DEM } & \\
\text { pi }=\text { kima-Pyu-su } & \text { oi-Pyu } & \text { kooha-u } \\
\text { RESTR =come-from-EMPH } & \text { DEM-from } & \text { pick.up-PFV }
\end{array}
$$

'Because when he lied, someone came along and stole it.'

(35) Mi'i usu peno'o usu.

$$
\begin{array}{llll}
\text { miPi } & \mathrm{u}-\mathrm{su} & \text { pino?o } & \mathrm{u}-\mathrm{su} \\
\text { QUOT } & \text { 3SG-NOM } & \text { as.well } & \text { 3SG-NOM }
\end{array}
$$

'And that's that story as well.'

(36) Kai kwaya nanateekwina usu oo.

kai kwaya na na-tïkwi-na u-su oo

NEG afar DISTR MID-tell-PTCP 3SG-NOM DEM

'That's not a story from too far back.'

(37) Oo ugau manina peno'o.

oo u-ga-u mani-na pino?o

DEM 3SG-GEN-FOC do-PTCP as.well

'That's his doings (that Coyote).'

\footnotetext{
${ }^{4}$ Translator also gave the additional comment, "It could mean that it's not far from being the real truth."
} 